\title{
Power Management and Reducing Routing Overhead and Delay in Wireless Sensor Network with Modified Ad- hoc on Demand Distance Vector Routing Protocol Using Threshold Power
}

\author{
Rubaina \\ Student \\ Department of Electronics Technology \\ Guru Nanak Dev University, Amritsar \\ Punjab-143005, India
}

\author{
Ravinder Singh Sawhney \\ Professor \\ Department of Electronics Technology \\ Guru Nanak Dev University, Amritsar \\ Punjab-143005, India
}

\begin{abstract}
Power management is necessary in Wireless Sensor Network because the nodes are very small in size having a small battery and that too of limited life. The routing algorithms for WSN should satisfy the features of energy consumption optimization and extension of network lifetime. We choose to modify AODV (Ad hoc On Demand Distance Vector) routing protocol, analyze this algorithm named as modified AODVTP (Threshold Power) and is highlighted in detail. The theme is that only those nodes are chosen as neighbors of nodes during RREQ (Route Request) phase that are directed towards destination. Also, threshold is set for all the nodes. If the power of the node is less than threshold, it will not acknowledge the Route Reply (RREP) packet. The alternate route with second lowest minimum distance will be selected to forward the packet. The behavioral analysis of the modified protocol is compared to AODV-TP and from the simulation this protocol turns out to be better in terms of power conservation.
\end{abstract}

\section{Keywords}

Wireless sensor network; power management; nodes; AODV

\section{INTRODUCTION}

Wireless Sensor Network consists of number of nodes which integrates sensors, transceiver of short range, processing subsystem and communicates with each other through wireless network. These networks are useful for a large number of applications including habitat [1], heath [2], pipeline [3], environment monitoring, agriculture [4], food safety, smart living and disaster management [5]. The nodes operate with batteries and these nodes are very small in size to accommodate large batteries. Also, it is not desirable to manually replace or recharge batteries. An efficient use of energy is a crucial concern in wireless sensor networks.

This paper enhances ad hoc on demand distance vector (AODV), which is one of the widely used reactive routing protocols, in order to reduce energy consumption and achieve the reliability of WSN [6]. In this proposed algorithm modified AODV-TP, the broadcast of RREQ (Route Request) packet has been modified. Only those nodes are chosen as neighbors of nodes during RREQ (Route Request) phase that are directed towards destination. Also, threshold value of each node is set; when the destination node replies back with respect to RREQ (Route request) packet each node acknowledges the RREP packet received. If battery power of any node is less than threshold value it will not acknowledge the received RREP packet and the packet will be forwarded through different route. In AODV-TP, only RREP phase is modified as described above. There is no change in RREQ phase. RREQ packets are forwarded to all neighbors.

\section{RELATED WORK}

Marina and Samir proposed Energy aware routing in Ad Hoc Networks in 2001 in which AODVEA selects a route with largest minimum residual energy and AODVM selects a route with the largest minimum residual energy and less hop count. There is no need to restart Route discovery if one of the links fails. But, there is need to uniquely identify each disjoint path on an end to end basis and to resolve issues related to ondemand multipath routing. [7]. Seema, Pinki and Rekha proposed AOMDV which also varies the transmission power between two nodes as per their distance [8]. It finds nodedisjoint paths by exploiting a particular property of flooding. Nishanthin. Rajkumar and Jayabhavan proposed EAODV protocol in 2013 with power boosted alternate paths. The number of hops travelled by the data and remaining hops is calculated using hop counter in case of detection of link or node failure. [9]. If number of hops travelled yet is greater than remaining hops then energy booster is enabled. Otherwise, the alternate path is selected. Divya, Manisha and Hari proposed AODV-TP (threshold Power) for MANETs [10].

\section{SIMULATION ALGORITHM DESIGN}

\subsection{Problem Formulation}

As AODV considers simple hop count metric to select the best path, it is not suitable for WSN. Therefore, the main purpose of this paper is to consider not only hop count but also energy metric and node lifetime when selecting the best path. 
The objective is to analyze, simulate and evaluate modified AODV-TP and to compare the performance of its power consumed, routing overhead and delay with AODV-TP.

\subsection{The Proposed Routing Algorithm}

The source node initiates the RREQ (Route Request) packet and forwards that to its neighbors which in turn forward to their neighbors and so on until the destination node receives the RREQ packet. Only those nodes are chosen as neighbors of nodes during RREQ (Route Request) phase that are directed towards destination that is $\mathrm{x}$ as well as $\mathrm{y}$ coordinate of the neighbors should be greater than that of the corresponding coordinates of source.

RREP is broadcasted by the destination node. In AODV, sending RREP (Route Reply) from destination to source node is similar to transmission of RREQ packet. However this leads to more energy consumption. The algorithm for transmission of RREP packet for modified AODV-TP is illustrated below:-

\section{1: Start}

2: Set threshold value of energy (say $4 \mathrm{~J}$ ) for each node.

3: For each node do

4: Obtain distance measurements of all the neighbors using distance formula

5: Choose the node with minimum distance.

6: if selected node=destination node then

7: Go to step 13

8: else if energy of selected node is greater than threshold then

9: Repeat step 3 for selected node

10: else

11: Backtrack to step 5 and choose another node from remaining set of nodes.

12: Repeat step 3 for this selected node

13: end if

14: end for

15: END

\section{Performance Evaluation}

\subsection{Simulation Setup}

MATLAB software has been used for the simulations of proposed algorithm due to its ease of node deployment and network set up. With the help of MATLAB 2013a, critical analysis of results is achieved. It has numerous built-in commands and math functions that help in mathematical calculations, generating plots and implementing algorithms.

In the beginning, 1000 nodes have been taken. Out of these nodes, 20 nodes have been randomly taken whose energy is set equal to $4 \mathrm{~J}$. The rest of the nodes have been provided $10 \mathrm{~J}$ of energy. The threshold value of energy is set for all the nodes (4J). Radio Energy Dissipation Model has been used by setting its parameters to calculate the remaining energy of nodes after these broadcast packets to their neighbors. The simulation is also done by taking different number of nodes.
Table 1. Simulation parameters

\begin{tabular}{|c|c|c|}
\hline $\begin{array}{c}\text { Sr. } \\
\text { No. }\end{array}$ & Parameters & Value \\
\hline 1 & Channel & $100 * 100$ sq. meter \\
\hline 2 & Simulation Area & $1000,200,500,800,1200$ \\
\hline 3 & $\begin{array}{c}\text { Number of } \\
\text { nodes }\end{array}$ & 1000 bits \\
\hline 4 & Packet size & $4 J$ \\
\hline 6 & Threshold & Matlab R2013a \\
\hline 7 & Simulator & \\
\hline
\end{tabular}

\subsection{Simulation Results and Analysis}

\subsubsection{Analysis of RREP Phase of Nodes}

The path followed for RREP phase has been shown with 'green' line. 'Before' represents the graphs for AODV-TP protocol and 'After' represents the graphs for the modified AODV-TP protocol.
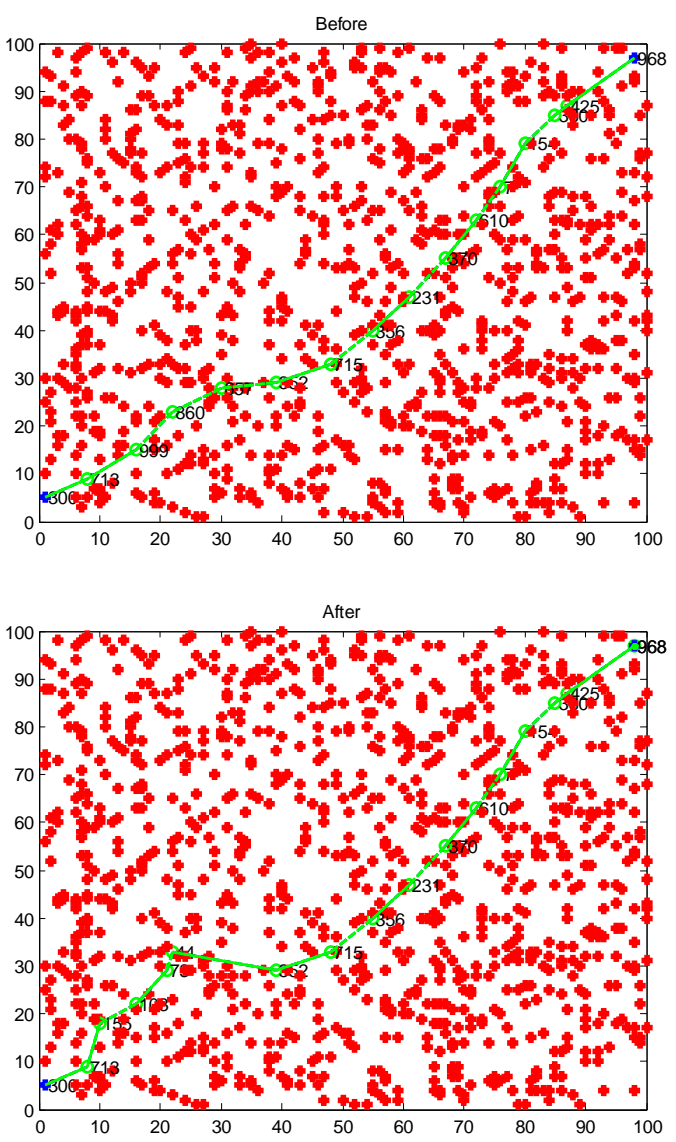

Fig 1: 1000 nodes 

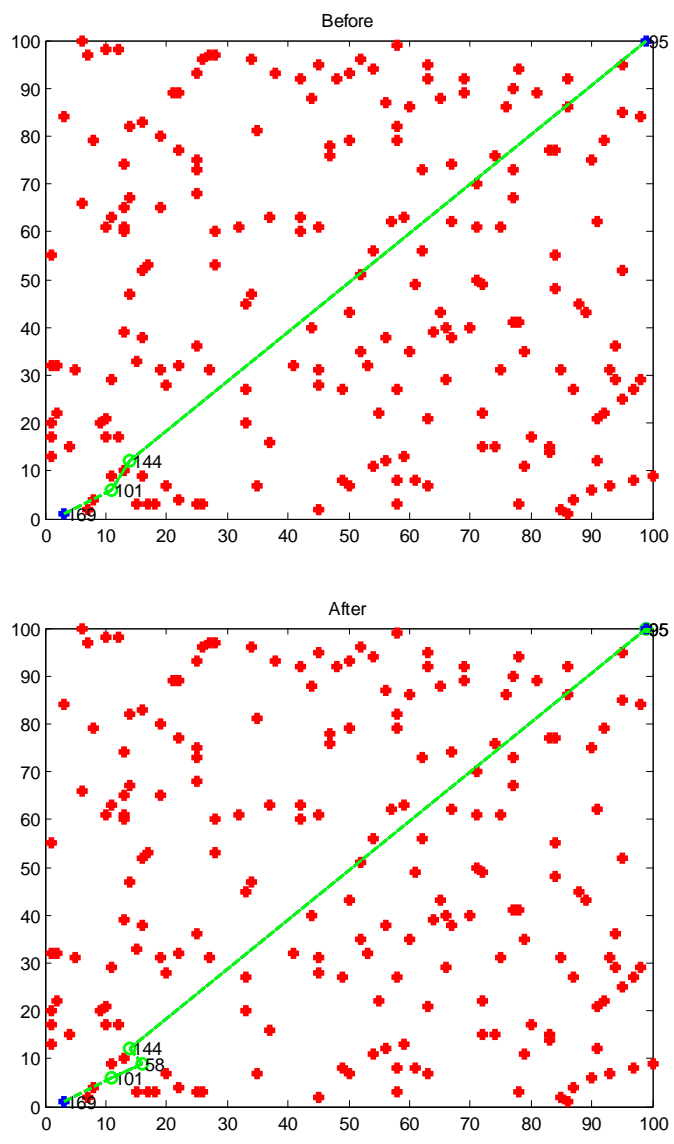

Fig 2: 200 nodes
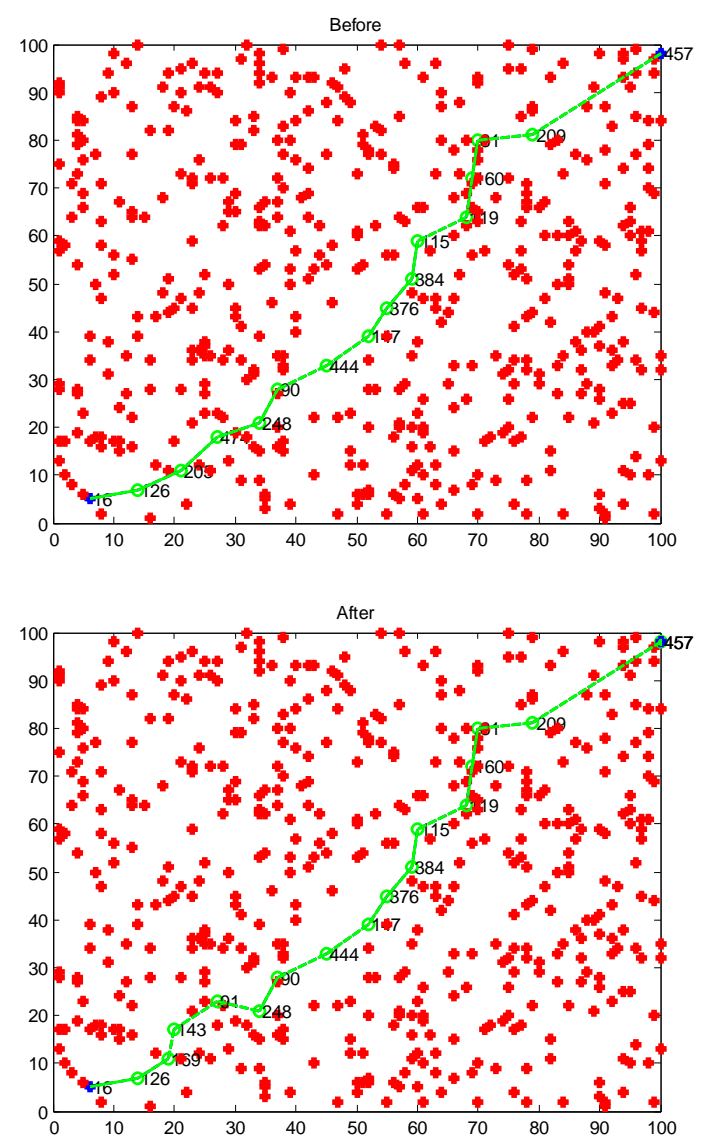

Fig 3: 500 nodes

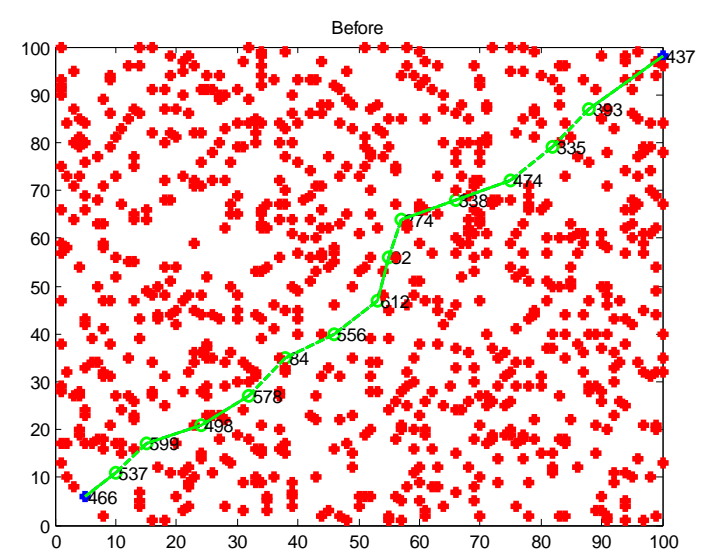




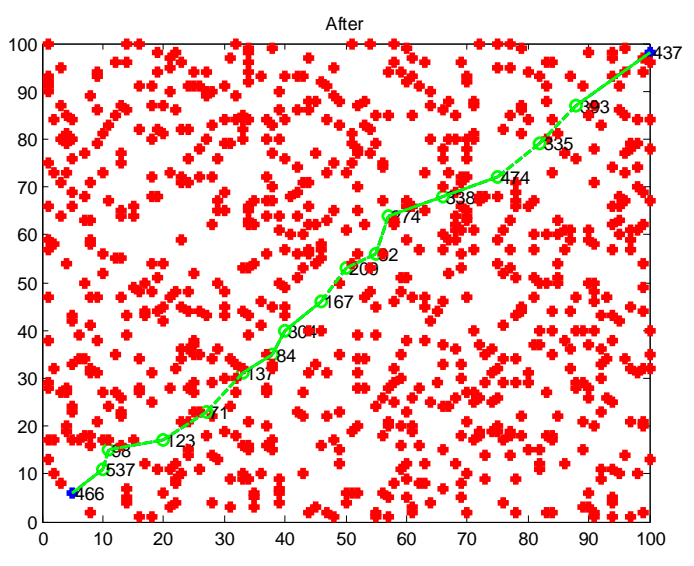

Fig 4: 800 nodes
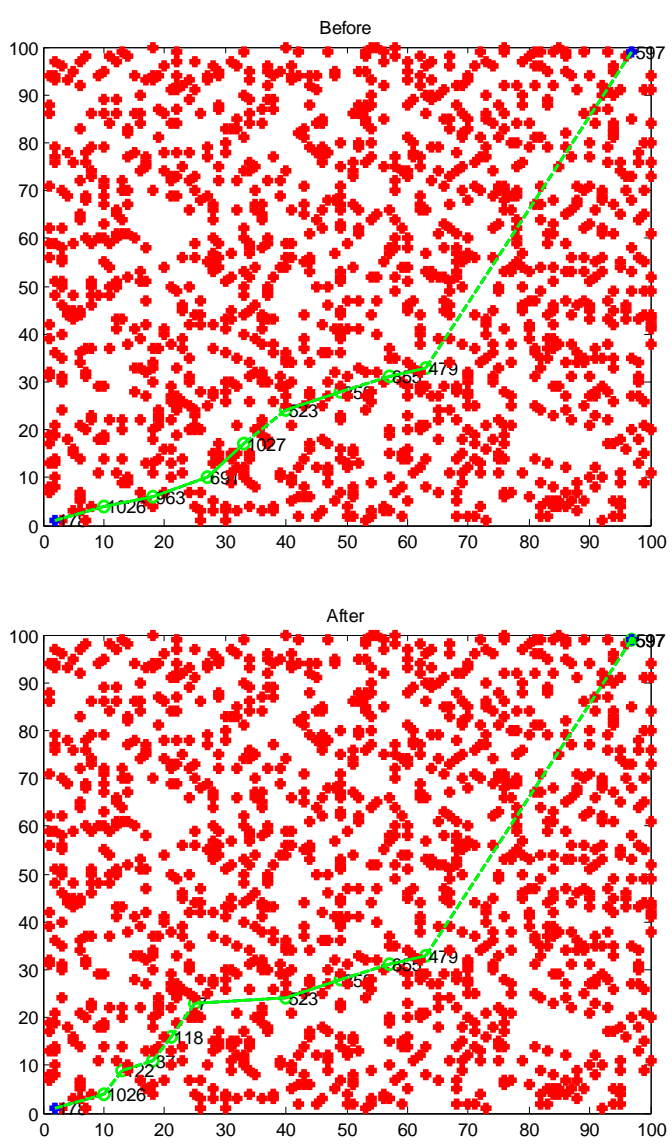

Fig 5: 1200 nodes

4.2.2 Power Comparison

It is observed that there is difference in the average remaining energy of the protocols.

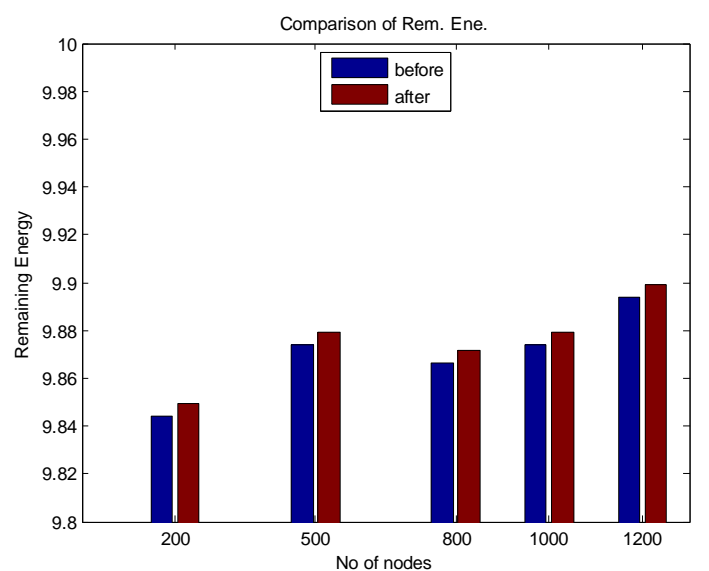

Fig 6: Comparison of remaining energy

The modified AODV-TP turns out to be more power efficient protocol than AODV-TP.

\subsubsection{Routing Overhead Comparison}

Routing overhead is the sum of all types of control packets sent during route discovery and route maintenance during data transfer. It also includes RREQ, RREP, and RERR (Route Error) messages also.

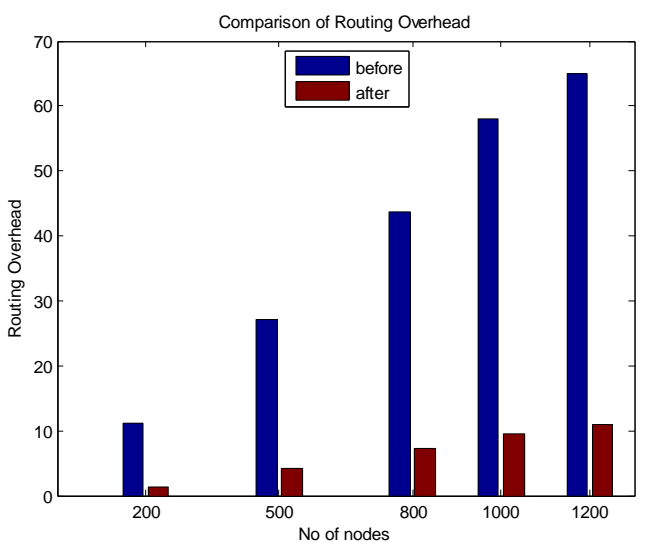

Fig 7: ComEparison of routing overhead

The routing overhead in the modified AODV-TP protocol decreases to a much extent.

\subsubsection{Delay Comparison}

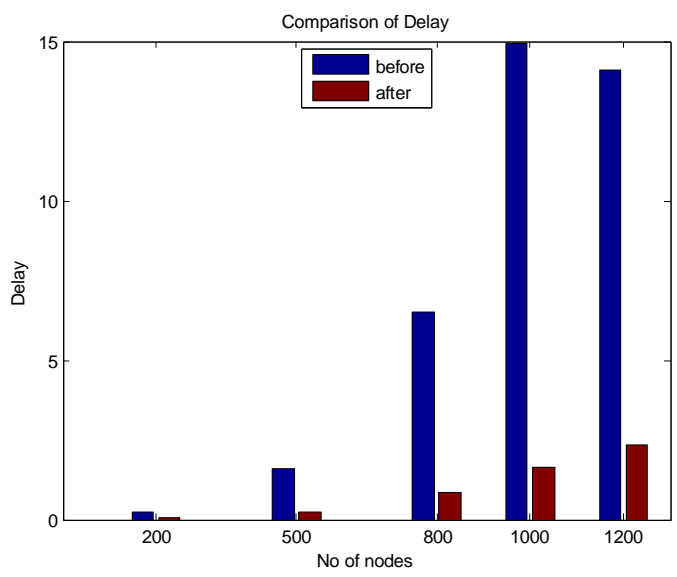

Fig 8: Comparison of delay 
It is observed that the delay in modified AODV-TP is less than AODV-TP.

Table 2. Results Comparison

\begin{tabular}{|c|c|c|c|}
\hline $\begin{array}{l}\text { No. of } \\
\text { Nodes }\end{array}$ & $\begin{array}{l}\text { Remaining } \\
\text { Energy }(\mathrm{J})\end{array}$ & $\begin{array}{l}\text { Routing } \\
\text { Overhead }\end{array}$ & $\begin{array}{l}\text { Delay } \\
(\mathrm{sec})\end{array}$ \\
\hline 200 & & & \\
\hline Before & 9.8439 & 11.8 & 0.2289 \\
\hline After & 9.8493 & 1.32 & 0.0738 \\
\hline 500 & & & \\
\hline Before & 9.8742 & 27.11 & 1.5865 \\
\hline After & 9.8792 & 4.24 & 0.2323 \\
\hline 800 & & & \\
\hline Before & 9.8666 & 43.68 & 6.502 \\
\hline After & 9.8715 & 7.26 & 0.8461 \\
\hline 1000 & & & \\
\hline Before & 9.8739 & 57.93 & 14.9442 \\
\hline After & 9.879 & 9.55 & 1.6512 \\
\hline 1200 & & & \\
\hline Before & 9.8941 & 64.92 & 14.0939 \\
\hline After & 9.8989 & 10.9 & 2.3541 \\
\hline
\end{tabular}

\section{CONCLUSION}

AODV-TP chooses that path in which the node participates in communication whose energy is greater than threshold. In modified AODV-TP, besides the above condition only those nodes are chosen as neighbors of node during RREQ phase that are directed towards destination. Comparison is done between the two in terms of average remaining energy of nodes, routing overhead and delay. It is observed that modified AODV-TP protocol is better in terms of power conservation.

\section{FUTURE WORK}

The simulations are required to be done for other parameters such as link capacity combined with the route selection logic so as to improve overall Quality of Service of wireless network. We can even simulate AODV protocol in IEEE 802.15.4 or Zigbee and analyze it using above performance metrics and compare the performance with IEEE 802.11.

\section{REFERENCES}

[1] Mainwaring, D. Culler, J. Polastre, R. Szewczyk, and J.Anderson, "Wireless sensor networks for habitat monitoring," ACM Int. Workshop Wireless Sensor Network Application, 2002, pp. 88-97

[2] S. Kim, S. Pakzad, D. Culler, J. Demmel, G. Fenves, S.Glaser, and M. Turon, "Health monitoring of civil infrastructures using wireless sensor networks," 6th Int Conf. Inf. Process. Sensor Network, New York, NY, 2007, pp. 254-263.

[3] I. Stoianov, L. Nachman, S. Madden, and T. Tokmouline, "Pipe net: A wireless sensor network for pipeline monitoring," 6th Int. Conf. Inf. Process. Sensor Netw. (IPSN), New York, NY, 2007, pp. 264-273

[4] A. Camilli, C. E. Cugnasca, A. M. Saraiva, A. R. Hirakawa, and P. L. P. Correa, "From wireless sensors to field mapping: Anatomy of an application for precision agriculture," Comput. Electron. Agric., vol. 58, no. 1, pp. 25-36, 2007

[5] Harminder Kaur, Ravinder Singh Sawhney, Navita Komal, "Wireless Sensor Networks for Disaster Management," International Journal of Advanced Research in Computer Engineering \& Technology Volume 1, Issue 5, July 2012,pp. 129-134

[6] C. Perkins, E. Royer, "Ad-Hoc On Demand Distance Vector Routing," the second IEEE workshops on Mobile Computer Systems and Applications, 1999. pp 90-100

[7] Marina, M. K., Das S, R.; "On Demand multi-path Distance Vector Routing in Ad hoc network,"IEEE International Conference on Network Protocols, 2001, pp.14-23

[8] Seema Verma , Pinki Nayak and Rekha Agarwal "Energy Efficient Routing in Mobile Adhoc Networks based on AODV Protocol" IJCSI International Journal of Computer Science Issues, Vol. 9, Issue 6, No 2, November 2012,pp.344-349

[9] C.Nishanthini, G.Rajkumar and G.N.Jayabhavani, "Enhanced Performance of AODV with Power Boosted Alternate Path," International Conference on Computer Communication and Informatics, Coimbatore, India, Jan. 04-06, 2013, pp.1-4

[10] Divya Sharma, Manisha Yadav and Hari Kumar" An ondemand energy efficient routing algorithm for mobile ad hoc networks" 